\title{
Bzip2 Algorithm
}

National Cancer Institute

\section{Source}

National Cancer Institute. bzip2 Algorithm. NCI Thesaurus. Code C80223.

An open source lossless data compression algorithm that compresses most files more effectively than the more traditional gzip or ZIP, but is slower. 